\title{
Group of Homography in Real Projective Plane
}

\author{
Roland Coghetto \\ Rue de la Brasserie 5 \\ 7100 La Louvière, Belgium
}

Summary. Using the Mizar system [2, we formalized that homographies of the projective real plane (as defined in [5]), form a group.

Then, we prove that, using the notations of Borsuk and Szmielew in 3]

"Consider in space $\mathbb{R P}^{2}$ points $P_{1}, P_{2}, P_{3}, P_{4}$ of which three points are not collinear and points $Q_{1}, Q_{2}, Q_{3}, Q_{4}$ each three points of which are also not collinear. There exists one homography $h$ of space $\mathbb{R P}^{2}$ such that $h\left(P_{i}\right)=Q_{i}$ for $i=1,2,3,4 . "$

(Existence Statement 52 and Existence Statement 53) [3]. Or, using notations of Richter [1]

"Let $[a],[b],[c],[d]$ in $\mathbb{R P}^{2}$ be four points of which no three are collinear and let $\left[a^{\prime}\right],\left[b^{\prime}\right],\left[c^{\prime}\right],\left[d^{\prime}\right]$ in $\mathbb{R P}^{2}$ be another four points of which no three are collinear, then there exists a $3 \times 3$ matrix $M$ such that $[M a]=\left[a^{\prime}\right],[M b]=\left[b^{\prime}\right],[M c]=\left[c^{\prime}\right]$, and $[M d]=\left[d^{\prime}\right] "$

Makarios has formalized the same results in Isabelle/Isar (the collineations form a group, lemma statement52-existence and lemma statement 53-existence) and published it in Archive of Formal Proof ${ }^{1}$ 10, 9].

MSC: 51N15 03B35

Keywords: projectivity; projective transformation; real projective plane; group of homography

MML identifier: ANPROJ_9, version: 8.1.05 5.40.1289

${ }^{1}$ http://isa-afp.org/entries/Tarskis_Geometry.shtml 


\section{Preliminaries}

From now on $i, n$ denote natural numbers, $r$ denotes a real number, $r_{1}$ denotes an element of $\mathbb{R}_{\mathrm{F}}, a, b, c$ denote non zero elements of $\mathbb{R}_{\mathrm{F}}, u, v$ denote elements of $\mathcal{E}_{\mathrm{T}}^{3}, p_{1}$ denotes a finite sequence of elements of $\mathbb{R}^{1}, p_{3}, u_{4}$ denote finite sequences of elements of $\mathbb{R}_{\mathrm{F}}, N$ denotes a square matrix over $\mathbb{R}_{\mathrm{F}}$ of dimension $3, K$ denotes a field, and $k$ denotes an element of $K$.

Now we state the propositions:

(1) $I_{\mathbb{R}_{\mathrm{F}}}^{3 \times 3}=\langle\langle 1,0,0\rangle,\langle 0,1,0\rangle,\langle 0,0,1\rangle\rangle$.

(2) $r_{1} \cdot N=r_{1} \cdot I_{\mathbb{R}_{\mathrm{F}}}^{3 \times 3} \cdot N$.

(3) If $r \neq 0$ and $u$ is not zero, then $r \cdot u$ is not zero.

ProOF: $r \cdot u \neq 0_{\mathcal{E}_{\mathrm{T}}^{3}}$ by [4, (52), (49)].

Let us consider elements $a_{11}, a_{12}, a_{13}, a_{21}, a_{22}, a_{23}, a_{31}, a_{32}, a_{33}$ of $\mathbb{R}_{\mathrm{F}}$ and a square matrix $A$ over $\mathbb{R}_{\mathrm{F}}$ of dimension 3 . Now we state the propositions:

(4) Suppose $A=\left\langle\left\langle a_{11}, a_{12}, a_{13}\right\rangle,\left\langle a_{21}, a_{22}, a_{23}\right\rangle,\left\langle a_{31}, a_{32}, a_{33}\right\rangle\right\rangle$. Then

(i) Line $(A, 1)=\left\langle a_{11}, a_{12}, a_{13}\right\rangle$, and

(ii) $\operatorname{Line}(A, 2)=\left\langle a_{21}, a_{22}, a_{23}\right\rangle$, and

(iii) $\operatorname{Line}(A, 3)=\left\langle a_{31}, a_{32}, a_{33}\right\rangle$.

(5) Suppose $A=\left\langle\left\langle a_{11}, a_{12}, a_{13}\right\rangle,\left\langle a_{21}, a_{22}, a_{23}\right\rangle,\left\langle a_{31}, a_{32}, a_{33}\right\rangle\right\rangle$. Then

(i) $A_{\square, 1}=\left\langle a_{11}, a_{21}, a_{31}\right\rangle$, and

(ii) $A_{\square, 2}=\left\langle a_{12}, a_{22}, a_{32}\right\rangle$, and

(iii) $A_{\square, 3}=\left\langle a_{13}, a_{23}, a_{33}\right\rangle$.

The theorem is a consequence of (4).

(6) Let us consider elements $a_{11}, a_{12}, a_{13}, a_{21}, a_{22}, a_{23}, a_{31}, a_{32}, a_{33}, b_{11}$, $b_{12}, b_{13}, b_{21}, b_{22}, b_{23}, b_{31}, b_{32}, b_{33}$ of $\mathbb{R}_{\mathrm{F}}$, and square matrices $A, B$ over $\mathbb{R}_{\mathrm{F}}$ of dimension 3. Suppose $A=\left\langle\left\langle a_{11}, a_{12}, a_{13}\right\rangle,\left\langle a_{21}, a_{22}, a_{23}\right\rangle,\left\langle a_{31}, a_{32}, a_{33}\right\rangle\right\rangle$ and $B=\left\langle\left\langle b_{11}, b_{12}, b_{13}\right\rangle,\left\langle b_{21}, b_{22}, b_{23}\right\rangle,\left\langle b_{31}, b_{32}, b_{33}\right\rangle\right\rangle$. Then $A \cdot B=\left\langle\left\langle a_{11}\right.\right.$. $\left.b_{11}+a_{12} \cdot b_{21}+a_{13} \cdot b_{31}, a_{11} \cdot b_{12}+a_{12} \cdot b_{22}+a_{13} \cdot b_{32}, a_{11} \cdot b_{13}+a_{12} \cdot b_{23}+a_{13} \cdot b_{33}\right\rangle$, $\left\langle a_{21} \cdot b_{11}+a_{22} \cdot b_{21}+a_{23} \cdot b_{31}, a_{21} \cdot b_{12}+a_{22} \cdot b_{22}+a_{23} \cdot b_{32}, a_{21} \cdot b_{13}+a_{22} \cdot\right.$ $\left.b_{23}+a_{23} \cdot b_{33}\right\rangle,\left\langle a_{31} \cdot b_{11}+a_{32} \cdot b_{21}+a_{33} \cdot b_{31}, a_{31} \cdot b_{12}+a_{32} \cdot b_{22}+a_{33} \cdot b_{32}\right.$, $\left.\left.a_{31} \cdot b_{13}+a_{32} \cdot b_{23}+a_{33} \cdot b_{33}\right\rangle\right\rangle$. The theorem is a consequence of (4) and (5).

(7) Let us consider elements $a_{11}, a_{12}, a_{13}, a_{21}, a_{22}, a_{23}, a_{31}, a_{32}, a_{33}, b_{1}, b_{2}$, $b_{3}$ of $\mathbb{R}_{\mathrm{F}}$, a matrix $A$ over $\mathbb{R}_{\mathrm{F}}$ of dimension $3 \times 3$, and a matrix $B$ over $\mathbb{R}_{\mathrm{F}}$ of dimension $3 \times 1$. Suppose $A=\left\langle\left\langle a_{11}, a_{12}, a_{13}\right\rangle,\left\langle a_{21}, a_{22}, a_{23}\right\rangle,\left\langle a_{31}, a_{32}, a_{33}\right\rangle\right\rangle$ and $B=\left\langle\left\langle b_{1}\right\rangle,\left\langle b_{2}\right\rangle,\left\langle b_{3}\right\rangle\right\rangle$. Then $A \cdot B=\left\langle\left\langle a_{11} \cdot b_{1}+a_{12} \cdot b_{2}+a_{13} \cdot b_{3}\right\rangle\right.$, 
$\left.\left\langle a_{21} \cdot b_{1}+a_{22} \cdot b_{2}+a_{23} \cdot b_{3}\right\rangle,\left\langle a_{31} \cdot b_{1}+a_{32} \cdot b_{2}+a_{33} \cdot b_{3}\right\rangle\right\rangle$. The theorem is a consequence of (4).

(8) Let us consider non zero elements $a, b, c$ of $\mathbb{R}_{F}$, and square matrices $M_{1}$, $M_{2}$ over $\mathbb{R}_{\mathrm{F}}$ of dimension 3 . Suppose $M_{1}=\langle\langle a, 0,0\rangle,\langle 0, b, 0\rangle,\langle 0,0, c\rangle\rangle$ and $M_{2}=\left\langle\left\langle\frac{1}{a}, 0,0\right\rangle,\left\langle 0, \frac{1}{b}, 0\right\rangle,\left\langle 0,0, \frac{1}{c}\right\rangle\right\rangle$. Then

(i) $M_{1} \cdot M_{2}=I_{\mathbb{R}_{\mathrm{F}}}^{3 \times 3}$, and

(ii) $M_{2} \cdot M_{1}=I_{\mathbb{R}_{\mathrm{F}}}^{3 \times 3}$.

The theorem is a consequence of (1).

(9) Let us consider non zero elements $a, b, c$ of $\mathbb{R}_{\mathrm{F}}$. Then $\langle\langle a, 0,0\rangle,\langle 0, b$, $0\rangle,\langle 0,0, c\rangle\rangle$ is an invertible square matrix over $\mathbb{R}_{\mathrm{F}}$ of dimension 3 . The theorem is a consequence of (8).

(10) (i) $[1,0,0]$ is not zero, and

(ii) $[0,1,0]$ is not zero, and

(iii) $[0,0,1]$ is not zero, and

(iv) $[1,1,1]$ is not zero.

(11) (i) $[1,0,0] \neq 0_{\mathcal{E}_{\mathrm{T}}^{3}}$, and

(ii) $[0,1,0] \neq 0_{\mathcal{E}_{\mathrm{T}}^{3}}$, and

(iii) $[0,0,1] \neq 0_{\mathcal{E}_{\mathrm{T}}^{3}}$, and

(iv) $[1,1,1] \neq 0_{\mathcal{E}_{\mathrm{T}}^{3}}$.

Proof: $[1,0,0] \neq[0,0,0]$ by [7, (2)]. $[0,1,0] \neq[0,0,0]$ by [7, (2)]. $[0,0$, $1] \neq[0,0,0]$ by $[7,(2)] .[1,1,1] \neq[0,0,0]$ by $[7,(2)]$.

(12) (i) $e_{1} \neq 0_{\mathcal{E}_{\mathrm{T}}^{3}}$, and

(ii) $e_{2} \neq 0_{\mathcal{E}_{\mathrm{T}}^{3}}$, and

(iii) $e_{3} \neq 0_{\mathcal{E}_{\mathrm{T}}^{3}}$.

Proof: $[1,0,0] \neq[0,0,0]$ by [7, $(2)] \cdot[0,1,0] \neq[0,0,0]$ by [7, (2)]. $[0,0$, $1] \neq[0,0,0]$ by [7, $(2)]$.

Let $n$ be a natural number. Note that $I_{\mathbb{R}_{\mathrm{F}}}^{n \times n}$ is invertible.

Let $M$ be an invertible square matrix over $\mathbb{R}_{\mathrm{F}}$ of dimension $n$. One can verify that $M^{\smile}$ is invertible.

Let $K$ be a field and $N_{1}, N_{2}$ be invertible square matrices over $K$ of dimension $n$. One can check that $N_{1} \cdot N_{2}$ is invertible. 


\section{Group of Homography}

From now on $N, N_{1}, N_{2}$ denote invertible square matrices over $\mathbb{R}_{\mathrm{F}}$ of dimension 3 and $P, P_{1}, P_{2}, P_{3}$ denote points of the projective space over $\mathcal{E}_{\mathrm{T}}^{3}$.

Now we state the propositions:

(13) (The homography of $\left.N_{1}\right)\left(\left(\right.\right.$ the homography of $\left.\left.N_{2}\right)(P)\right)=$ (the homography of $\left.N_{1} \cdot N_{2}\right)(P)$.

Proof: Consider $u_{12}, v_{12}$ being elements of $\mathcal{E}_{\mathrm{T}}^{3}, u_{8}$ being a finite sequence of elements of $\mathbb{R}_{F}, p_{12}$ being a finite sequence of elements of $\mathbb{R}^{1}$ such that $P=$ the direction of $u_{12}$ and $u_{12}$ is not zero and $u_{12}=u_{8}$ and $p_{12}=N_{1} \cdot N_{2} \cdot u_{8}$ and $v_{12}=\operatorname{M} 2 \mathrm{~F}\left(p_{12}\right)$ and $v_{12}$ is not zero and (the homography of $\left.N_{1} \cdot N_{2}\right)(P)=$ the direction of $v_{12}$. Consider $u_{2}, v_{2}$ being elements of $\mathcal{E}_{\mathrm{T}}^{3}, u_{6}$ being a finite sequence of elements of $\mathbb{R}_{\mathrm{F}}, p_{2}$ being a finite sequence of elements of $\mathbb{R}^{1}$ such that $P=$ the direction of $u_{2}$ and $u_{2}$ is not zero and $u_{2}=u_{6}$ and $p_{2}=N_{2} \cdot u_{6}$ and $v_{2}=\mathrm{M} 2 \mathrm{~F}\left(p_{2}\right)$ and $v_{2}$ is not zero and (the homography of $\left.N_{2}\right)(P)=$ the direction of $v_{2}$. Consider $u_{1}, v_{1}$ being elements of $\mathcal{E}_{\mathrm{T}}^{3}, u_{7}$ being a finite sequence of elements of $\mathbb{R}_{\mathrm{F}}, p_{1}$ being a finite sequence of elements of $\mathbb{R}^{1}$ such that (the homography of $\left.N_{2}\right)(P)=$ the direction of $u_{1}$ and $u_{1}$ is not zero and $u_{1}=u_{7}$ and $p_{1}=N_{1} \cdot u_{7}$ and $v_{1}=$ $\operatorname{M} 2 \mathrm{~F}\left(p_{1}\right)$ and $v_{1}$ is not zero and (the homography of $N_{1}$ )((the homography of $\left.\left.N_{2}\right)(P)\right)=$ the direction of $v_{1}$. Consider $a$ being a real number such that $a \neq 0$ and $u_{2}=a \cdot u_{12}$. Consider $b$ being a real number such that $b \neq 0$ and $u_{1}=b \cdot v_{2} \cdot v_{1}=\left\langle\left(N_{1} \cdot\left\langle u_{7}\right\rangle^{\mathrm{T}}\right)_{1,1},\left(N_{1} \cdot\left\langle u_{7}\right\rangle^{\mathrm{T}}\right)_{2,1},\left(N_{1} \cdot\left\langle u_{7}\right\rangle^{\mathrm{T}}\right)_{3,1}\right\rangle$ by [1, (1), (40)]. $v_{2}=\left\langle\left(N_{2} \cdot\left\langle u_{6}\right\rangle^{\mathrm{T}}\right)_{1,1},\left(N_{2} \cdot\left\langle u_{6}\right\rangle^{\mathrm{T}}\right)_{2,1},\left(N_{2} \cdot\left\langle u_{6}\right\rangle^{\mathrm{T}}\right)_{3,1}\right\rangle$ by [1, (1), (40)]. $v_{12}=\left\langle\left(N_{1} \cdot N_{2} \cdot\left\langle u_{8}\right\rangle^{\mathrm{T}}\right)_{1,1},\left(N_{1} \cdot N_{2} \cdot\left\langle u_{8}\right\rangle^{\mathrm{T}}\right)_{2,1},\left(N_{1} \cdot N_{2} \cdot\left\langle u_{8}\right\rangle^{\mathrm{T}}\right)_{3,1}\right\rangle$ by [1. (1), (40)]. Reconsider $v_{6}=v_{2}$ as a finite sequence of elements of $\mathbb{R}_{\mathrm{F}}$. Reconsider $i_{4}=\frac{1}{b}$ as a real number. $v_{6}=i_{4} \cdot u_{1}$ by [4, (49), (52)]. Reconsider $l_{11}=\operatorname{Line}\left(N_{2}, 1\right)(1), l_{12}=\operatorname{Line}\left(N_{2}, 1\right)(2), l_{13}=\operatorname{Line}\left(N_{2}, 1\right)(3)$, $l_{21}=\operatorname{Line}\left(N_{2}, 2\right)(1), l_{22}=\operatorname{Line}\left(N_{2}, 2\right)(2), l_{23}=\operatorname{Line}\left(N_{2}, 2\right)(3), l_{31}=$ Line $\left(N_{2}, 3\right)(1), l_{32}=\operatorname{Line}\left(N_{2}, 3\right)(2), l_{33}=\operatorname{Line}\left(N_{2}, 3\right)(3)$ as an element of $\mathbb{R}_{\mathrm{F}} \cdot N_{2 \square, 1}=\left\langle l_{11}, l_{21}, l_{31}\right\rangle$ and $N_{2 \square, 2}=\left\langle l_{12}, l_{22}, l_{32}\right\rangle$ and $N_{2 \square, 3}=\left\langle l_{13}, l_{23}\right.$, $\left.l_{33}\right\rangle$ by [1, (1), (45)]. The direction of $v_{1}=$ the direction of $v_{12}$ by [5, (7)], [1, (45)], [5, (93)], [7, (8)].

(14) (The homography of $\left.I_{\mathbb{R}_{F}}^{3 \times 3}\right)(P)=P$.

(15) (i) (the homography of $N)\left(\left(\right.\right.$ the homography of $\left.\left.N^{\smile}\right)(P)\right)=P$, and

(ii) (the homography of $\left.N^{\smile}\right)(($ the homography of $N)(P))=P$.

The theorem is a consequence of (13) and (14).

(16) If (the homography of $N)\left(P_{1}\right)=($ the homography of $N)\left(P_{2}\right)$, then $P_{1}=$ $P_{2}$. The theorem is a consequence of (15). 
(17) Let us consider a non zero element $a$ of $\mathbb{R}_{\mathrm{F}}$. Suppose $a \cdot I_{\mathbb{R}_{\mathrm{F}}}^{3 \times 3}=N$. Then (the homography of $N)(P)=P$.

The functor EnsHomography3 yielding a set is defined by the term

(Def. 1) the set of all the homography of $N$ where $N$ is an invertible square matrix over $\mathbb{R}_{\mathrm{F}}$ of dimension 3 .

One can check that EnsHomography3 is non empty.

Let $h_{1}, h_{2}$ be elements of EnsHomography3. The functor $h_{1} \circ h_{2}$ yielding an element of EnsHomography3 is defined by

(Def. 2) there exist invertible square matrices $N_{1}, N_{2}$ over $\mathbb{R}_{\mathrm{F}}$ of dimension 3 such that $h_{1}=$ the homography of $N_{1}$ and $h_{2}=$ the homography of $N_{2}$ and $i t=$ the homography of $N_{1} \cdot N_{2}$.

Now we state the propositions:

(18) Let us consider elements $h_{1}, h_{2}$ of EnsHomography3. Suppose $h_{1}=$ the homography of $N_{1}$ and $h_{2}=$ the homography of $N_{2}$. Then the homography of $N_{1} \cdot N_{2}=h_{1} \circ h_{2}$. The theorem is a consequence of (13).

(19) Let us consider elements $x, y, z$ of EnsHomography3. Then $(x \circ y) \circ z=$ $x \circ(y \circ z)$. The theorem is a consequence of (18).

The functor BinOpHomography3 yielding a binary operation on

EnsHomography3 is defined by

(Def. 3) for every elements $h_{1}, h_{2}$ of EnsHomography3, it $\left(h_{1}, h_{2}\right)=h_{1} \circ h_{2}$.

The functor GroupHomography3 yielding a strict multiplicative magma is defined by the term

(Def. 4) 〈EnsHomography3, BinOpHomography3 $\rangle$.

Note that GroupHomography3 is non empty, associative, and group-like.

Now we state the propositions:

(20) $\quad \mathbf{1}_{\text {GroupHomography3 }}=$ the homography of $I_{\mathbb{R}_{\mathrm{F}}}^{3 \times 3}$.

(21) Let us consider elements $h, g$ of GroupHomography3, and invertible square matrices $N, N_{10}$ over $\mathbb{R}_{\mathrm{F}}$ of dimension 3 . Suppose $h=$ the homography of $N$ and $g=$ the homography of $N_{10}$ and $N_{10}=N^{\smile}$. Then $g=h^{-1}$. The theorem is a consequence of (20).

\section{Main Results}

The functors: Dir100, Dir010, Dir001, and Dir111 yielding points of the projective space over $\mathcal{E}_{\mathrm{T}}^{3}$ are defined by terms

(Def. 5) the direction of $[1,0,0]$,

(Def. 6) the direction of $[0,1,0]$, 
(Def. 7) the direction of $[0,0,1]$,

(Def. 8) the direction of $[1,1,1]$, respectively. Now we state the proposition:

(22) (i) Dir100 $\neq$ Dir010, and

(ii) $\operatorname{Dir} 100 \neq$ Dir001, and

(iii) Dir100 $\neq$ Dir111, and

(iv) $\operatorname{Dir} 010 \neq$ Dir001, and

(v) $\operatorname{Dir} 010 \neq \operatorname{Dir} 111$, and

(vi) Dir001 $\neq$ Dir111.

Let $a$ be a non zero element of $\mathbb{R}_{\mathrm{F}}$. Let us consider $N$. Note that $a \cdot N$ is invertible as a square matrix over $\mathbb{R}_{\mathrm{F}}$ of dimension 3 .

(23) Let us consider a non zero element $a$ of $\mathbb{R}_{\mathrm{F}}$. Then (the homography of $\left.a \cdot N_{1}\right)(P)=\left(\right.$ the homography of $\left.N_{1}\right)(P)$. The theorem is a consequence of (2), (13), and (17).

(24) Suppose $P_{1}, P_{2}$ and $P_{3}$ are not collinear. Then there exists an invertible square matrix $N$ over $\mathbb{R}_{\mathrm{F}}$ of dimension 3 such that

(i) (the homography of $N)\left(P_{1}\right)=\operatorname{Dir} 100$, and

(ii) (the homography of $N)\left(P_{2}\right)=$ Dir010, and

(iii) (the homography of $N)\left(P_{3}\right)=\operatorname{Dir} 001$.

Proof: Consider $u_{1}$ being an element of $\mathcal{E}_{\mathrm{T}}^{3}$ such that $u_{1}$ is not zero and $P_{1}=$ the direction of $u_{1}$. Consider $u_{2}$ being an element of $\mathcal{E}_{\mathrm{T}}^{3}$ such that $u_{2}$ is not zero and $P_{2}=$ the direction of $u_{2}$. Consider $u_{3}$ being an element of $\mathcal{E}_{\mathrm{T}}^{3}$ such that $u_{3}$ is not zero and $P_{3}=$ the direction of $u_{3}$. Reconsider $p_{3}=u_{1}, q_{1}=u_{2}, r_{2}=u_{3}$ as a finite sequence of elements of $\mathbb{R}_{\mathrm{F}}$. Consider $N$ being a square matrix over $\mathbb{R}_{\mathrm{F}}$ of dimension 3 such that $N$ is invertible and $N \cdot p_{3}=\mathrm{F} 2 \mathrm{M}\left(e_{1}\right)$ and $N \cdot q_{1}=\mathrm{F} 2 \mathrm{M}\left(e_{2}\right)$ and $N \cdot r_{2}=\mathrm{F} 2 \mathrm{M}\left(e_{3}\right)$. (The homography of $\left.N\right)\left(P_{1}\right)=\operatorname{Dir} 100$ by [8, (22), (1)], [6. (22)], [5, (75)]. (The homography of $N)\left(P_{2}\right)=\operatorname{Dir} 010$ by [8, (22), (1)], [6. (22)], [5, (75)]. (The homography of $N)\left(P_{3}\right)=\operatorname{Dir001}$ by [8, (22), (1)], [6, (22)], [5, (75)].

(25) Let us consider non zero elements $a, b, c$ of $\mathbb{R}_{\mathrm{F}}$. Suppose $N=\langle\langle a, 0,0\rangle$, $\langle 0, b, 0\rangle,\langle 0,0, c\rangle\rangle$. Then

(i) (the homography of $N)(\operatorname{Dir} 100)=\operatorname{Dir} 100$, and

(ii) (the homography of $N)(\operatorname{Dir} 010)=$ Dir010, and

(iii) (the homography of $N)($ Dir001) $=$ Dir001. 
Proof: (The homography of $N)(\operatorname{Dir} 100)=$ Dir100 by (12), [8, (22), (1)], [7. (8), (2)]. (The homography of $N)(\operatorname{Dir010})=$ Dir010 by (12), [8, (22), (1)], [7, (8), (2)]. (The homography of $N)(\operatorname{Dir} 001)=\operatorname{Dir001}$ by (12), 8, $(22),(1)],[7,(8),(2)]$.

Let us consider a point $P$ of the projective space over $\mathcal{E}_{\mathrm{T}}^{3}$.

(26) There exist elements $a, b, c$ of $\mathbb{R}_{\mathrm{F}}$ such that

(i) $P=$ the direction of $[a, b, c]$, and

(ii) $a \neq 0$ or $b \neq 0$ or $c \neq 0$.

(27) Suppose Dir100, Dir010 and $P$ are not collinear and Dir100, Dir001 and $P$ are not collinear and Dir010, Dir001 and $P$ are not collinear. Then there exist non zero elements $a, b, c$ of $\mathbb{R}_{\mathrm{F}}$ such that $P=$ the direction of $[a, b$, $c]$. The theorem is a consequence of (26).

(28) Let us consider non zero elements $a, b, c, i_{1}, i_{2}, i_{3}$ of $\mathbb{R}_{\mathrm{F}}$, a point $P$ of the projective space over $\mathcal{E}_{\mathrm{T}}^{3}$, and an invertible square matrix $N$ over $\mathbb{R}_{\mathrm{F}}$ of dimension 3. Suppose $P=$ the direction of $[a, b, c]$ and $i_{1}=\frac{1}{a}$ and $i_{2}=\frac{1}{b}$ and $i_{3}=\frac{1}{c}$ and $N=\left\langle\left\langle i_{1}, 0,0\right\rangle,\left\langle 0, i_{2}, 0\right\rangle,\left\langle 0,0, i_{3}\right\rangle\right\rangle$. Then (the homography of $N)(P)=$ the direction of $[1,1,1]$.

Proof: Consider $u, v$ being elements of $\mathcal{E}_{\mathrm{T}}^{3}, u_{4}$ being a finite sequence of elements of $\mathbb{R}_{\mathrm{F}}, p$ being a finite sequence of elements of $\mathbb{R}^{1}$ such that $P=$ the direction of $u$ and $u$ is not zero and $u=u_{4}$ and $p=N \cdot u_{4}$ and $v=$ $\operatorname{M} 2 \mathrm{~F}(p)$ and $v$ is not zero and (the homography of $N)(P)=$ the direction of $v$. [a,b,c] is not zero by [7, (4)], [1, (78)]. Consider $d$ being a real number such that $d \neq 0$ and $u=d \cdot[a, b, c]$. Reconsider $d_{1}=d \cdot a, d_{2}=d \cdot b, d_{3}=d \cdot c$ as an element of $\mathbb{R}_{\mathrm{F}} \cdot v=\left[i_{1} \cdot d_{1}, i_{2} \cdot d_{2}, i_{3} \cdot d_{3}\right]$ by [1, (45)].

(29) Let us consider a point $P$ of the projective space over $\mathcal{E}_{\mathrm{T}}^{3}$. Suppose Dir100, Dir010 and $P$ are not collinear and Dir100, Dir001 and $P$ are not collinear and Dir010, Dir001 and $P$ are not collinear. Then there exist non zero elements $a, b, c$ of $\mathbb{R}_{\mathrm{F}}$ such that for every invertible square matrix $N$ over $\mathbb{R}_{\mathrm{F}}$ of dimension 3 such that $N=\langle\langle a, 0,0\rangle,\langle 0, b, 0\rangle,\langle 0,0, c\rangle\rangle$ holds (the homography of $N)(P)=\operatorname{Dir111}$. The theorem is a consequence of (27) and (28).

(30) Let us consider points $P_{1}, P_{2}, P_{3}, P_{4}$ of the projective space over $\mathcal{E}_{\mathrm{T}}^{3}$. Suppose $P_{1}, P_{2}$ and $P_{3}$ are not collinear and $P_{1}, P_{2}$ and $P_{4}$ are not collinear and $P_{1}, P_{3}$ and $P_{4}$ are not collinear and $P_{2}, P_{3}$ and $P_{4}$ are not collinear. Then there exists an invertible square matrix $N$ over $\mathbb{R}_{\mathrm{F}}$ of dimension 3 such that

(i) (the homography of $N)\left(P_{1}\right)=$ Dir100, and

(ii) (the homography of $N)\left(P_{2}\right)=$ Dir010, and 
(iii) (the homography of $N)\left(P_{3}\right)=$ Dir001, and

(iv) (the homography of $N)\left(P_{4}\right)=\operatorname{Dir} 111$.

The theorem is a consequence of (24), (29), (9), (25), and (13).

(31) Let us consider points $P_{1}, P_{2}, P_{3}, P_{4}, Q_{1}, Q_{2}, Q_{3}, Q_{4}$ of the projective space over $\mathcal{E}_{\mathrm{T}}^{3}$. Suppose $P_{1}, P_{2}$ and $P_{3}$ are not collinear and $P_{1}, P_{2}$ and $P_{4}$ are not collinear and $P_{1}, P_{3}$ and $P_{4}$ are not collinear and $P_{2}, P_{3}$ and $P_{4}$ are not collinear and $Q_{1}, Q_{2}$ and $Q_{3}$ are not collinear and $Q_{1}, Q_{2}$ and $Q_{4}$ are not collinear and $Q_{1}, Q_{3}$ and $Q_{4}$ are not collinear and $Q_{2}, Q_{3}$ and $Q_{4}$ are not collinear. Then there exists an invertible square matrix $N$ over $\mathbb{R}_{\mathrm{F}}$ of dimension 3 such that

(i) (the homography of $N)\left(P_{1}\right)=Q_{1}$, and

(ii) (the homography of $N)\left(P_{2}\right)=Q_{2}$, and

(iii) (the homography of $N)\left(P_{3}\right)=Q_{3}$, and

(iv) (the homography of $N)\left(P_{4}\right)=Q_{4}$.

The theorem is a consequence of (30), (15), and (13).

\section{REFERENCES}

[1] Grzegorz Bancerek and Krzysztof Hryniewiecki. Segments of natural numbers and finite sequences. Formalized Mathematics, 1(1):107-114, 1990.

[2] Grzegorz Bancerek, Czesław Byliński, Adam Grabowski, Artur Korniłowicz, Roman Matuszewski, Adam Naumowicz, Karol Pąk, and Josef Urban. Mizar: State-of-the-art and beyond. In Manfred Kerber, Jacques Carette, Cezary Kaliszyk, Florian Rabe, and Volker Sorge, editors, Intelligent Computer Mathematics, volume 9150 of Lecture Notes in Computer Science, pages 261-279. Springer International Publishing, 2015. ISBN 978-3319-20614-1. doi 10.1007/978-3-319-20615-8_17.

[3] Karol Borsuk and Wanda Szmielew. Foundations of Geometry. North Holland, 1960.

[4] Czesław Byliński. The sum and product of finite sequences of real numbers Formalized Mathematics, 1(4):661-668, 1990.

[5] Roland Coghetto. Homography in $\mathbb{R P}^{2}$. Formalized Mathematics, 24(4):239-251, 2016. doi:10.1515/forma-2016-0020.

[6] Agata Darmochwał. The Euclidean space. Formalized Mathematics, 2(4):599-603, 1991.

[7] Kanchun, Hiroshi Yamazaki, and Yatsuka Nakamura. Cross products and tripple vector products in 3-dimensional Euclidean space. Formalized Mathematıcs, 11(4):381-383, 2003.

[8] Wojciech Leończuk and Krzysztof Prażmowski. A construction of analytical projective space. Formalized Mathematics, 1(4):761-766, 1990.

[9] Timothy James McKenzie Makarios. The independence of Tarski's Euclidean Axiom. Archive of Formal Proofs, October 2012.

[10] Timothy James McKenzie Makarios. A mechanical verification of the independence of Tarski's Euclidean Axiom. Victoria University of Wellington, New Zealand, 2012. Master's thesis.

[11] Jürgen Richter-Gebert. Perspectives on projective geometry: a guided tour through real and complex geometry. Springer Science \& Business Media, 2011.

Received March 17, 2017 